Meta

Journal des traducteurs

Translators' Journal

\title{
Étude diachronique et évolution du concept de résonance en physique
}

\section{Ali Mouhouche et Abdelkrim El-Hajjami}

Volume 58, numéro 2, août 2013

URI : https://id.erudit.org/iderudit/1024182ar

DOI : https://doi.org/10.7202/1024182ar

Aller au sommaire du numéro

\section{Éditeur(s)}

Les Presses de l’Université de Montréal

\section{ISSN}

0026-0452 (imprimé)

1492-1421 (numérique)

Découvrir la revue

\section{Citer cet article}

Mouhouche, A. \& El-Hajjami, A. (2013). Étude diachronique et évolution du concept de résonance en physique. Meta, 58(2), 430-448.

https://doi.org/10.7202/1024182ar

\section{Résumé de l'article}

Dans la présente étude diachronique, nous examinons d'un côté l'origine et les significations du verbe résonner et de ses dérivés, notamment le terme résonance, et de l'autre, l'évolution, d'après les manuels de physique à différentes dates, du concept scientifique de résonance en physique des ondes. Nous nous demandons si la terminologie exprime et désigne toujours le phénomène physique dont la signification s'est élargie, généralisée, modifiée et complexifiée au fil du temps. Pour cela, nous utilisons essentiellement des dictionnaires et des manuels de physique anciens. Le terme résonance, exprimant à l'origine un phénomène naturel ressenti surtout par l'ouïe, s'est d'abord limité au domaine de l'acoustique, dont il garde l'estampille jusqu'à maintenant. Il s'est par la suite élargi à divers types d'ondes et à diverses dimensions de la nature, des particules élémentaires de la matière jusqu'aux planètes. De plus, il véhicule les idées d'accord et de transfert d'énergie inexistantes initialement. Ces nouvelles réalités scientifiques rendent sa traduction ambiguë et sa compréhension difficile. Tenant compte de ce principal résultat, nous proposons une dénomination qui évite la confusion tout en étant plus adaptée à sa signification scientifique actuelle. 


\title{
TERMINOLOGIE ET LINGUISTIQUE
}

\section{Étude diachronique et évolution du concept de résonance en physique}

\author{
ALI MOUHOUCHE \\ École Normale Supérieure, Kouba; École Nationale Supérieure d'Agronomie, Alger, Algérie \\ amouhouche@yahoo.fr
}

\author{
ABDELKRIM EL-HAJJAMI \\ École Normale Supérieure de Fès, Maroc \\ alhajjami@yahoo.com
}

\section{RÉSUMÉ}

Dans la présente étude diachronique, nous examinons d'un côté l'origine et les significations du verbe résonner et de ses dérivés, notamment le terme résonance, et de l'autre, l'évolution, d'après les manuels de physique à différentes dates, du concept scientifique de résonance en physique des ondes. Nous nous demandons si la terminologie exprime et désigne toujours le phénomène physique dont la signification s'est élargie, généralisée, modifiée et complexifiée au fil du temps. Pour cela, nous utilisons essentiellement des dictionnaires et des manuels de physique anciens. Le terme résonance, exprimant à l'origine un phénomène naturel ressenti surtout par l'ouïe, s'est d'abord limité au domaine de l'acoustique, dont il garde l'estampille jusqu'à maintenant. II s'est par la suite élargi à divers types d'ondes et à diverses dimensions de la nature, des particules élémentaires de la matière jusqu'aux planètes. De plus, il véhicule les idées d'accord et de transfert d'énergie inexistantes initialement. Ces nouvelles réalités scientifiques rendent sa traduction ambiguë et sa compréhension difficile. Tenant compte de ce principal résultat, nous proposons une dénomination qui évite la confusion tout en étant plus adaptée à sa signification scientifique actuelle.

\begin{abstract}
In this diachronic study we first investigate the changes that occurred throughout the history to the verb to resound and its derivatives, in particular the term resonance, and then the evolution of the scientific concept of resonance in physics of the waves. We wonder if the terminology always indicates and expresses the physical phenomenon whose significance was broadened, generalized, modified, and became more complex. For this purpose, we use various dictionaries and historical handbooks of physics. The term of resonance, first expressing a natural phenomenon felt particularly by hearing, was initially limited to the field of acoustics, of which it keeps the mark until now. It was extended thereafter to several types of waves and to various dimensions of natural things, from elementary particles of matter to planets in space. Moreover, it conveys the notions of chord and transfer of energy, which it didn't initially. These new scientific realities make it ambiguous for traducing and more difficult to understand. Taking account of this main result, we propose a denomination that avoids confusion and is more adapted to the concept's current scientific significance.
\end{abstract}

\section{MOTS-CLÉS/KEYWORDS}

diachronie, traduction, résonance, didactique, histoire diachrony, translation, resonance, didactics, history 


\section{Introduction}

Il est reconnu que la terminologie diachronique reste encore un domaine relativement peu exploré par les études terminologiques (Dury 1999: 485). Pourtant, elle pourrait être d'un grand apport par des réflexions originales et riches, notamment sur des points de vue linguistiques ou historiques, parfois les deux, relatifs aux concepts et à leur évolution. En plus de sa contribution en traduction et en linguistique, elle peut également éclairer des aspects scientifiques disciplinaires ou épistémologiques utiles aux historiens des sciences et aux didacticiens. Dury (2006: 110) écrit que «les études de type diachronique posent de véritables questions terminologiques et apportent des solutions nouvelles sur la manière de les résoudre». Une des situations problématiques auxquelles l'étude diachronique pourrait apporter des éléments de réponse est décrite également par cette auteure dans le passage suivant:

Seule la terminologie diachronique peut montrer à quel point l'évolution d'un concept peut être complexe et inattendue, et lourde de conséquences pour la dénomination qu'il porte. Il y a très souvent inadéquation entre l'évolution d'une notion et celle de sa dénomination, qui ne varie que très rarement, même si le concept ne cesse de se modifier, de se complexifier. (Dury 1999: 485-486)

Les études diachroniques ne constituent donc pas une activité marginale de la terminologie, mais offrent une approche nouvelle des termes, des concepts et de leurs relations.

Mais pourquoi la présente étude diachronique de la résonance en physique des ondes, et que peut-elle nous apporter?

Il existe très peu de travaux sur l'analyse diachronique des concepts de sciences physiques. Nous n'en avons pas trouvé sur le concept de résonance en physique des ondes. Le phénomène de résonance est important et omniprésent dans le domaine de la physique des vibrations, des rayonnements et des échanges ondes-matière. Il fait partie des phénomènes ondulatoires et il est enseigné dès la classe de terminale des lycées pour certaines filières, puis à l'université dans le cadre des programmes de physique générale. Notons quelques-unes de ses particularités les plus frappantes:

- la résonance concerne quasiment tous les types d'ondes;

- elle concerne des objets de dimensions très variées, des particules élémentaires aux planètes;

- elle pourrait aussi bien être recherchée, pour en tirer des informations inaccessibles par d'autres moyens, ou évitée en raison de son aspect destructeur dû à l'accumulation de grande quantité d'énergie vibratoire.

La résonance est un phénomène qui intervient aussi en acoustique architecturale, dans la distorsion du son, son absorption ou le niveau de confusion des sonorités d'une salle. Nous lui connaissons une utilisation dans le langage courant également.

Dans le cadre d'une recherche plus vaste en didactique des sciences, nous avions abouti à l'existence d'un obstacle verbal qui rend difficile la compréhension du phénomène de résonance par les étudiants (Mouhouche et El-Hajjami 2010). Ces derniers font très souvent référence au son ou au bruit dans leurs définitions de la résonance, ils font des confusions sur sa phénoménologie et l'amalgament avec le phénomène d'amplification sonore. De plus, la résonance n'est pas comprise par l'étudiant comme un phénomène basé sur l'influence d'un objet sur un autre, mais comme une propriété de l'objet résonant. Ces difficultés montrent un déficit dans la conceptualisation 
et sont des répercussions de transpositions didactiques de manuels et de livres spécialisés lors de traductions, d'adaptations ou d'élaborations de textes pour le cours ou les activités d'apprentissage. Nous pensons que cette étude diachronique du concept de résonance apportera des éléments d'explication à cette situation et aboutira à une proposition de remédiation possible.

Au sein de la didactique, le regard diachronique et celui de l'histoire des sciences en général restent encore marginaux, et presque inexistants, affirment Celotti et Musacchio (2004: 265), qui ont étudié leurs implications dans les processus de formation, notamment universitaires. Madrane, Talbi, et al. (2008) font un constat analogue. Ce manque est aussi l'une des raisons pour lesquelles nous entreprenons de réaliser la présente étude. Le parallèle entre l'évolution historique du sens du terme résonance et les modifications du concept scientifique qu'il représente pourrait révéler des éléments complémentaires utiles à sa traduction, ce qui se répercuterait sur son étude didactique. Nous comptons également alimenter notre quête de difficultés et d'obstacles, notamment de type épistémologique, qui se dresseraient sur le chemin de la construction et de l'apprentissage du concept de résonance.

La question qui se pose est la suivante: est-ce que la terminologie exprime et désigne toujours le phénomène physique dont la signification s'est élargie et généralisée, modifiée et complexifiée en désignant de plus en plus d'objets et d'évènements de la réalité?

La résonance est un phénomène naturel, à l'origine détectable par les sens, qui a évolué vers un phénomène physique beaucoup plus important. Nous soutenons, en guise d'hypothèse que le terme résonance, avec sa signification première et sa connotation sonore issues du langage courant, ne peut plus désigner de façon efficace un concept qui n'a cessé d'évoluer et de s'étendre à divers objets, et à toutes les dimensions physiques, des particules microscopiques de la matière jusqu'aux astres. En effet, les sciences physiques ont progressé et traversé différents paradigmes du passé (Kuhn 2001), de la physique ancienne à la physique quantique et moderne, en passant par la physique classique. Dès lors, compte tenu de ces progrès, un terme apparu à une époque pourrait-il rester suffisamment significatif au fil du temps? Dury (1999: 487) affirme que «[...] très souvent les concepts scientifiques évoluent sans que les dénominations qui les désignent en soit modifiées pour autant». À ce sujet, Conceição (1999: 36) soutient que le risque de déformation d'un concept est grand en cours de transmission des connaissances, et d'autant plus important que les connaissances $\mathrm{du}$ récepteur sur le domaine sont faibles. C'est le cas des situations d'enseignement où l'apprenant (récepteur) est censé découvrir et apprendre au sujet d'un nouveau phénomène qu'il ignorait.

Après quelques considérations théoriques qui nous seront utiles pour la compréhension du développement du présent travail, nous mènerons une étude terminologique historique en utilisant plusieurs dictionnaires. Nous montrerons ensuite l'évolution conceptuelle qu'a connue la résonance à travers des manuels de sciences physiques anciens, accompagnant, en principe, les évolutions scientifiques relatives au concept en question. Nous mettrons enfin en parallèle les deux approches, dans une dernière partie, pour en tirer des résultats à notre questionnement et conclurons par quelques propositions d'ordre terminologique et didactique.

Tout en mettant en exergue l'intérêt de l'étude terminologique diachronique pour les sciences et leur histoire, cette recherche pourrait bénéficier autant au 
domaine de la traduction, notamment de textes scientifiques, qu'à celui de l'enseignement-apprentissage du concept de résonance en physique des ondes.

\section{La recherche terminologique: de la conception wüsterienne à la théorie sociocognitiviste}

Une synthèse, effectuée principalement à partir de travaux sur la recherche terminologique, nous permet de présenter l'essentiel des fondements théoriques, d'introduire et d'expliciter succinctement les outils conceptuels utilisés dans la présente recherche et de choisir notre posture.

Pour l'école de Vienne (Wüster 1991, cité dans Temmerman 2000a; Felber 1987) la discipline de la terminologie se réduit à un ensemble de principes de normalisation. Idéalement, pour cette école traditionaliste ou classique, un terme unique est attribué à chaque notion ou concept ${ }^{1}$, et ce, à titre permanent. Les notions et les termes sont étudiés de façon synchronique, tout en considérant la relation entre notion et terme comme arbitraire (Temmerman 2000a: 58). Mais ces principes ont des limites qui ont été identifiées: ils ne suffisent pas pour établir une terminographie réaliste, Temmerman (1998b) en a fait la démonstration pour les disciplines scientifiques qui portent sur les sciences de la vie. Elle écrit: «On a pu constater l'influence du langage comme instrument cognitif et comme donnée sociologique sur le processus continu de compréhension", ajoutant que «[c]eci nous mène à établir une théorie de la terminologie descriptive fondée sur des principes sociocognitifs» (Temmerman 2000a: 58).

La théorie sociocognitive de la terminologie, développée par Temmerman (2000a: 59), introduit les unités de compréhension (units of understanding) ou unités de connaissance. Ces unités représentent des savoirs et sont aussi des unités d'interprétation. Temmerman pose le principe que ces unités sont en évolution permanente et que, selon les cas, les périodes chronologiques concernées peuvent être essentielles à la compréhension de l'unité. Conceição écrit:

L'étude sociocognitive de la terminologie donne la possibilité de suivre l'évolution du sens des termes, c'est-à-dire qu'elle peut se servir de la terminologie diachronique pour expliquer la compréhension et l'interprétation du monde. (Conceição 2005: 41)

Le terme est alors considéré à la fois comme signe linguistique et dénomination d'un concept utilisé pour exprimer des connaissances (Conceição 2005: 233), en se définissant explicitement en référence à un domaine du savoir dont il est solidaire. Par le savoir qu'il exprime, le terme a le pouvoir d'établir des ponts entre ce qui est su et ce qui est nouveau, de même qu'avec d'autres concepts.

Dans notre travail, nous nous plaçons dans cette perspective, qui donne la possibilité de suivre l'évolution du sens du terme (Temmerman 2000b: 37, cité dans Conceição 2005: 41). Dans cette optique «[1]a conceptualisation découle de l'expérience et de la compréhension du monde», témoigne également Clas (2001: 584) dans son compte rendu de l'ouvrage de Temmerman. La polysémie, qui «est entre autres le résultat de la réflexion humaine sur le monde» ou, d'un point de vue sémasiologique, «le résultat de l'évolution sémantique du Langage» ainsi que la synonymie, doivent être décrites (Temmerman 2000a: 59, 61).

Les principaux concepts guides apparus dans ce regard théorique et dans la problématique décrite auparavant sont ceux de terme, de notion (ou concept), de conceptualisation, de cognition et de compréhension, de traduction et de diachronie. 


\section{Analyse diachronique du concept de résonance}

Dans cette analyse nous examinons l'origine et les significations du verbe résonner et de ses dérivés, notamment le terme résonance, puis l'évolution, d'après des manuels de physique publiés à différentes dates, du concept scientifique de résonance en physique des ondes.

\subsection{Résonner et ses dérivés: aspects métalexicographiques}

En guise de repères terminologiques, regardons, en premier lieu, les dates d'apparition et les définitions des termes résonner, résonance, résonant et résonateur dans le vocabulaire. Nous adoptons une démarche chronologique et utilisons pour cela essentiellement des dictionnaires anciens: le Thresor de la langue françoyse (Nicot $\left.1606^{2}\right)$, le Dictionnaire de l'Académie Française $\left(1694^{3} ; D A F\right)$, le Dictionnaire étymologique de la langue française (Brachet $1870^{4}$ ), le Trésor de la langue française informatisé (2012; TLFi') et le Französisches Etymologisches Wörterbuch (FEW) de Walther von Wartburg (Wartburg 1948 ${ }^{6}$ ). Les dictionnaires Le Nouveau Petit Robert (20017 ; NPR), Oxford Dictionary $\left(2012^{8}\right)$, Collins French-English Dictionary et Collins English Dictionary $\left(2012^{9}\right)$ ont été aussi consultés.

Résonner est apparu dès l'an 1155 dans le FEW (Wartburg 1948) et l'an 1160 dans le TLFi). Il provient du latin resonare (Wartburg 1948: 305; Nicot 1606: 563 ; Brachet 1870 : 464; TLFi), qui signifie: rendre un grand son, beaucoup de son, renvoyer le son par réflexion, renvoyer un écho, retentir (du bruit de...), s'emplir de bruit, d'échos, de résonances (lieu). Il a le sens de sonner, tinter, retentir, comme dans l'expression des pas résonnent sur la chaussée.

L'origine du substantif résonnance remonte à 1365 selon le TLFi et à 1374 selon le FEW. Il était alors utilisé dans le domaine musical avec le sens de: retentissement, prolongation de la durée d'un son, prolongement ou amplification des sons dans certains milieux sonores. Il devint, en 1532, la propriété de renvoyer le son, de l'accroître, que possèdent certains objets, telle une cloche, ou certains milieux, telle une voûte (Wartburg 1948: 305a, 305b).

En 1765, il désigne la propriété qu'a un corps d'entrer en vibration quand il est soumis à une excitation convenable (Wartburg 1948: 305b). L'idée de renforcement de l'intensité vibratoire ou sonore est donc apparue, appliquée aux caisses d'instruments à cordes dans les expressions: caisse de résonance (enceinte fermée où se produisent des phénomènes de résonance); la bouche et les fosses nasales forment des cavités de résonance pour les sons du langage.

Le TLFi précise qu'avant 1878 , on écrivait résonnance (avec deux « $\mathrm{n} »$ ), et que le terme provient du latin resonantia, qui signifie écho, ce que confirme le $F E W$, pour qui ce terme ne serait pas un emprunt, mais bien une création nouvelle. En effet, on retrouve l'orthographe résonnance, avec deux n, dans divers documents historiques. Citons entre autres les Annales de chimie et de physique (Antoine 1849: 191), et un ouvrage édité en 1877 par Pietro Blaserna (Blaserna 1877), dans lequel sont consignés les termes résonnance (34), résonner (44) et résonnateur (46), toujours avec deux «n».

Le terme résonnant est utilisé en 1538 et caractérisait ce qui renvoie le son, qui produit beaucoup de son (Wartburg 1948), ce qui est retentissant, sonore ou ce qui 
est le siège d'un phénomène de résonance (TLFi). Les expressions données en appui sont: système résonant, chambre résonante.

Le terme résonnateur est utilisé en science à partir de 1868, lorsque l'Académie des Sciences publia un compte rendu sur le tube résonnateur (TLFi), et est défini comme un appareil servant à analyser les sons, puis comme un appareil pour mettre en évidence les ondes électriques (Wartburg 1948). Plus généralement, il s'agit d'un appareil à l'intérieur duquel peut se produire un phénomène de résonance, un milieu matériel capable d'entrer en vibration sous l'influence d'un excitateur. C'est le sens indiqué par les expressions: résonateur acoustique, résonateur électrique, résonateur de Helmholtz ou de Hertz ou résonateur nucléaire. Ce qui était système résonant est devenu résonateur, ou l'on peut dire que dans le système résonant l'on reconnaissait l'existence d'un résonateur.

Pour revenir au terme résonance, il prit en 1746 le sens de "propriété d'un corps d'entrer en vibration» dans le TLFi, et en 1765 , le FEW ajouta «... lorsqu'il est soumis à une excitation convenable» (Wartburg 1948: XX). Le Nouveau Petit Robert précise qu'il est utilisé dans le domaine scientifique à partir du milieu du XIX ${ }^{\mathrm{e}}$ siècle, de l'an 1862 plus exactement, et défini alors comme l'« [a]ugmentation de l'amplitude d'un système physique en vibration lorsque la vibration excitatrice se rapproche d'une fréquence naturelle de ce système» (NPR 2001). Il apparaît, avec ces changements, l'idée nouvelle de l'intervention d'un élément excitateur (inexistant auparavant) pour provoquer la résonance.

Nous retenons qu'à l'origine de l'utilisation du terme résonance (1365 ou 1374), celui-ci concernait essentiellement le son et faisait référence aux traitements ou aux transformations que subissait un son émis de même qu'à ses effets (tels que son prolongement, la génération d'autres sons dans un milieu, etc.). Autrement dit, la faculté de résonner était attribuée au son lui-même et non à d'autres objets. C'est à partir du milieu du $\mathrm{XIX}^{\mathrm{e}}$ siècle que la résonance a cessé d'être considérée comme une simple propriété rattachée au son et décrivant son comportement. L'augmentation de l'amplitude, le système physique en vibration, la vibration excitatrice et la fréquence naturelle (appelée plus tard fréquence propre) sont quatre notions nouvelles qui, dans leur existence simultanée, modifient radicalement la signification de la résonance en physique tout en lui donnant un sens nouveau, du moins plus général, s'appliquant à des situations de résonance plus vastes. Désormais la résonance n'est plus un attribut du son en soi, mais caractérise plutôt un système physique en vibration. Le phénomène s'étend à d'autres objets possibles mis en résonance grâce à une vibration excitatrice, qui en constitue la cause et qui peut avoir une origine autre que les ondes sonores. La résonance est sortie de la sonorisation ${ }^{10}$ pour prendre une nouvelle signification.

\subsection{Définitions historiques du concept de résonance dans les manuels}

Nous examinons dans cette partie les différentes définitions données au terme de résonance depuis le milieu du $\mathrm{XIX}^{\mathrm{e}}$ siècle, notamment dans les livres destinés à l'enseignement. Des ouvrages et des manuels de physique ainsi que des textes anciens nous servent de corpus. Dans ce qui suit, nous suivrons une démarche chronologique. Les manuels anciens utilisés sont édités à partir de 1847. 


\subsubsection{XIX ${ }^{e}$ siècle: sonorisation de la résonance et objets sonores}

\subsubsection{Milieu du XIX ${ }^{e}$ siècle: une première signification (renforcement ou réflexion d'un son)}

Peclet, en 1847, dans son Traité élémentaire de physique, fait une description détaillée du phénomène, qu'il appelle «communication des mouvements vibratoires des corps, à travers les liquides et à travers l'air» (Peclet 1847: 347). Il utilise l'expression corps ébranlé pour désigner ce qui est appelé maintenant le résonateur. Citons deux passages significatifs pour notre sujet:

Pour qu'un corps rigide soit mis en vibration sonore, il suffit d'imprimer à une partie quelconque du corps une vive agitation: elle se communique bientôt à la masse entière [...] Il y a un mode d'ébranlement plus favorable que tous les autres. (Peclet 1847:330)

Les cordes sont mises en vibration par l'air lorsqu'il transmet des sons harmoniques des leurs. C'est encore ainsi que les sons de l'orgue font souvent frémir et résonner les vitres qui sont susceptibles de vibrer à l'unisson. [...] Les caisses résonnent sous l'influence des cordes dont elles renforcent le son. (Peclet 1847: 347)

Nous notons l'adjectif sonore, qui caractérise la vibration dans la première citation, et nous remarquons que le verbe résonner est lié par une conjonction de coordination à frémir, comme pour le compléter ou se poser en synonyme, dans la deuxième. L'expression les caisses résonnent, dans la dernière phrase, a un sens de produisent un son.

Il ressort donc que chez Peclet (1847):

- le phénomène est appelé communication des mouvements vibratoires des corps ou renforcement de son; le substantif résonance n'est pas utilisé;

- le verbe résonner est utilisé, mais dans le sens de provoquer ou renforcer des vibrations sonores;

- les objets décrits comme mis en vibration sonore sont les cordes, les caisses, les vitres et des objets rigides;

- la fréquence propre, qui est un paramètre très important dans le phénomène de résonance, est reconnue implicitement grâce aux sons harmoniques (dans les cordes vibrantes) et à la reconnaissance du mode d'ébranlement plus favorable que tous les autres.

On retrouve le concept de résonance, cité cette fois nommément, et défini de façon différente, tout en conservant la notion de prolongement d'un son, chez Boutet de Monvel en 1863. Dans son Cours de physique, dans un chapitre réservé à l'acoustique, nous trouvons un sous-chapitre intitulé Échos et résonnance, où l'auteur explique l'origine de la résonance ainsi que sa signification. Il écrit:

Échos et résonances - C’est la réflexion du son qui donne naissance au phénomène des échos et des résonances. L'impression produite par un son sur l'organe de l'ouïe ne s'éteint pas immédiatement avec la cause qui l'a provoquée; elle persiste encore pendant un certain temps, très court il est vrai, car il n'est guère que d'un dixième de seconde. (Boutet de Monvel 1863: 641)

Et à la page suivante:

Mais supposons que le mur s'éloigne de telle sorte que la différence $(\mathrm{AC}+\mathrm{CB}-\mathrm{AB}) / 337$ soit à peu près $1 / 10$ de seconde. Alors le son réfléchi vient prolonger, et peut peut-être doubler la durée du son direct. C'est alors qu'on dit qu'il y a résonance. Si le mur 
s'éloigne davantage, alors le son réfléchi devient nettement distinct du son direct, la résonance est devenue un écho. (Boutet de Monvel 1863: 642)

La résonance n'était nullement la mise en vibration d'un résonateur (tympan) par un excitateur (onde sonore), c'était plutôt l'arrivée à l'oreille d'un son "réfléchi qui vient alors prolonger et peut-être doubler la durée du son direct», lui faisant ainsi écho ${ }^{11}$. Cette définition du terme résonance, rattachée à la réflexion et à l'écho du son, est confirmée par d'autres ouvrages dont nous donnons quelques exemples ci-dessous.

Dans son Traité élémentaire de physique théorique et expérimentale Daguin écrit:

Résonnance - Quand un son réfléchi empiète sur le son direct, comme cela a lieu lorsque le centre phonocamptique est peu éloigné, il y a résonnance; le son direct est renforcé par sa coïncidence partielle avec le son réfléchi, mais il devient confus, par la prolongation qu'y apporte ce dernier. (Daguin 1862: 479)

Edouard Fournié, en 1866, écrit également:

Résonnance - Lorsque la réflexion se fait dans un espace plus étendu que les tuyaux sonores et ne mesurant pas plus cependant de 34 mètres, comme dans les appartements par exemple, le son n'acquiert pas les caractères qu'il revêt dans les tuyaux sonores sous l'influence de la réflexion, mais il est renforcé: il y a résonnance. (Fournié 1866: 19)

Notons que la distance de 34 mètres correspond approximativement à la distance parcourue en un dixième de seconde par le son, sachant que sa vitesse dans l'air ambiant est d'environ $340 \mathrm{~m} / \mathrm{s}$.

En 1877, dans un livre contenant également des textes sur les résonateurs de Helmholtz, Blaserna, après avoir expliqué ce qu'est la réflexion d'une onde sur un obstacle, écrit: «Les deux effets les plus importants de la réflexion sont la résonnance et l'écho» (Blaserna 1877: 34-35).

La résonance est donc définie dans les manuels cités précédemment, qui vont de 1847 à 1877, soit comme un renforcement d'un son, soit comme un effet de la réflexion du son, au même titre que l'écho. La résonance est alors le prolongement d'un son, tandis que l'écho en est la reproduction après un temps dépassant le dixième de seconde.

\subsubsection{Fin $d u X X^{e}$ siècle: introduction de la notion de transmission (ou communication) de vibrations}

Dans son Cours de physique de l'école polytechnique (tome 3), dans le chapitre intitulé Acoustique, Jamin parle de résonance en insistant sur des exemples sonores tirés de l'étude de la théorie mathématique de l'élasticité (1887: 164-169). La résonance acoustique est appelée renforcement des sons. Le phénomène de résonance autre qu'acoustique est décrit par l'expression transmission ou communication de vibrations. Dans le cas de cordes voisines, il écrit: «Si les deux cordes sont d'accord, chaque impulsion continue l'effet de la précédente, et la vibration se transmet et s'exagère » (Jamin 1887: 166). Au sujet d'une cloche que l'on met en oscillations, nous lisons:

[s]upposons que l'on tire pendant un temps très court la corde d'une cloche; on lui imprime aussitôt des oscillations qui se continuent pendant longtemps. Si l'on répète l'impulsion plusieurs fois successivement, il peut arriver deux cas extrêmes ou que chacune d'elles soit discordante avec le mouvement imprimé par la précédente, alors elle le détruit, ou bien qu'elle soit concordante, et alors elle l'augmente. Dans ce cas, l'amplitude des oscillations de la cloche grandit peu à peu jusqu'à devenir considérable. (Jamin 1887: 165-166) 
En revanche, à la page 158, la résonance est décrite sans être nommée telle que nous la définirons plus loin, comme un cas particulier de vibrations forcées où il y a égalité de fréquences de l'excitateur et du résonateur. Cependant, nous relevons le fait que le terme résonance n'est pas utilisé pour nommer le phénomène, et que celuici semble être différent, pour l'auteur, dans le cas de la corde et celui de la cloche.

En résumé, nous pouvons dire que dès les années 1880, le phénomène de résonance a été appelé transmission de vibration. La résonance concernait encore les vibrations sonores plus que les autres formes de vibrations. Les objets concernés étaient des corps sonores, cordes ou objets vibrants qui résonnaient. Vers la fin du $\mathrm{XIX}^{\mathrm{e}}$ siècle, la définition de la résonance a commencé à contenir les idées d'augmentation de l'amplitude du corps ébranlé et de fréquence propre.

La deuxième moitié du $\mathrm{XIx}^{\mathrm{e}}$ siècle a donc vu, sur le plan de la transposition didactique dans les manuels:

- la persistance de la sonorisation de la résonance par la référence au son plus quaux vibrations mécaniques en général;

- le maintien de la préoccupation autour d'objets sonores comme objets vibrants;

- le rattachement de l'étude du phénomène aux chapitres sur l'acoustique et non pas aux chapitres sur les mouvements vibratoires ou sur les ondes;

- un premier changement conceptuel intervenu à partir des années 80 de ce siècle.

\subsubsection{Début du $x x^{e}$ siècle: nouveaux objets résonants et généralisation $d u$ concept}

Dès la fin du XIX ${ }^{\mathrm{e}}$ siècle, l'influence des travaux sur la résonance de certains savants, notamment Helmholtz, Hertz et Tesla, commençait à se répercuter sur les contenus des manuels d'enseignement. Chappuis et Berget, dans leurs Leçons de physique générale (tome 3, portant la mention "Rédigé suivant le programme de la licence ès sciences physiques») et, plus particulièrement, dans un cours portant sur l'acoustique, écrivent:

Il est facile de constater que, quand un diapason, donnant $u t_{1}$ par exemple, entre en vibration, tout autre diapason voisin donnant $u t_{1}$ vibre aussi, quoiqu'il n'ait pas été excité directement. Un tel diapason est le type du résonateur, c'est-à-dire d'un instrument susceptible de vibrer à l'unisson d'une note donnée et, par suite, d'en renforcer le son. (Chappuis et Berget 1892: 68)

Dans les pages 333 et 334 du même ouvrage, l'expérience de Hertz est décrite, mais seuls les termes excitateur et résonateur sont utilisés, sans celui de résonance. Helmholtz est cité en acoustique à la page 68.

En 1911, Chappuis et Lamotte (1911: 48) utilisent le terme résonance dans le tome IV (réservé aux ondes électriques) en parlant de l'expérience de Hertz, dans le sens admis aujourd'hui. Les auteurs parlent également de la résonance électrique RLC à la page 291 du tome II (publié en 1920) réservé à l'électricité. Par ailleurs, ils décrivent une expérience de pendule oscillant entretenu électriquement (dans la partie «instrument de mesure» du tome I, 1924: 71), donc en situation de résonance grâce à l'apport de l'énergie d'un circuit électrique, sans faire recours à la notion de résonance.

En optique, Paul Drude, dans son Précis d'optique (tome 1) (1911: 316), tente d'expliquer les phénomènes de modification de la lumière survenant, dans les milieux troubles, par l'absorption d'un rayonnement par résonance, et en donne des résultats 
dans le tome 2, décrivant ceux-ci comme «absolument conformes aux phénomènes généraux de résonance» (Drude 1911: 174).

$\mathrm{Au}$ début $\mathrm{du} \mathrm{xx}^{\mathrm{e}}$ siècle, l'acceptation de la résonance comme phénomène se produisant par l'effet d'un excitateur sur un résonateur est la principale avancée dans la transposition didactique. Elle constitue un premier pas vers la généralisation du concept de résonance. L'extension de la notion de résonance aux ondes hertziennes, aux ondes optiques et aux ondes électriques, alors qu'elle était auparavant restreinte aux ondes sonores, en est une conséquence notable. Malgré cela, la résonance orbitale en astronomie et la résonance mécanique, toutes deux bien connues à cette époque, ne figurent toujours pas dans les programmes d'enseignement, et la référence au son et à la musique a persisté dans quelques ouvrages. Par exemple, en 1926 le Dictionnaire pratique et historique de la musique définit la résonance comme étant la production de sons harmoniques à un son principal d'une source sonore (Brenet 1926: 389 ${ }^{12}$ ). Cela est compréhensible pour un ouvrage consacré à la musique, mais entretient quand même la conception «sonore» de la résonance.

Notons que Heisenberg, en 1926, utilisa le mot résonance pour la première fois en mécanique quantique dans ses travaux sur les systèmes d'oscillateurs couplés, et que Pauling, dans ses recherches sur la nature de la liaison chimique vers 1930, introduisit la résonance en chimie.

\subsubsection{Le reste $d u X x^{e}$ siècle}

Durant la deuxième moitié du $\mathrm{xx}^{\mathrm{e}}$ siècle, la plupart des ouvrages intégrèrent le sens de résonance véhiculant les quatre composantes, à savoir: l'excitateur, le résonateur, la fréquence propre et l'augmentation de l'amplitude du résonateur en situation de résonance, donc implicitement le transfert d'énergie de l'excitateur vers le résonateur. Les ouvrages contemporains s'approchent tous de cette conception de la résonance, chacun privilégiant cependant un aspect au détriment d'un autre suivant le sujet ou les applications prévues par l'auteur. Si une définition insiste sur l'échange d'énergie qui se produit pendant un phénomène de résonance, une autre évoque la vibration à la fréquence propre, tandis qu'une autre encore met en exergue l'augmentation démesurée de l'amplitude de vibration du résonateur et que certaines insistent sur le couplage ou sur l'amortissement.

En ce qui concerne la résonance dans l'atome, dans la molécule (vibration ou rotation de molécules) ou dans le noyau ${ }^{13}$, les manuels de physique générale n'en parlent que très rarement, considérant probablement qu'il revient aux chimistes ou aux cours de physique avancés (physique atomique, physique nucléaire, physique des particules, etc.) de le faire. De même, nous n'avons pas rencontré de référence, dans les manuels de cours de physique, à la résonance orbitale ou à la résonance de Laplace $\left(\right.$ en astronomie $\left.{ }^{14}\right)$.

\subsection{Phénoménologie de la résonance, stabilité ou emballement du résonateur}

Au cours de l'histoire, il y a eu passage d'un fait empirique, détectable par les sens (audible au début, puis visible à travers les cordes), vers un phénomène physique général, ce phénomène ayant donné lieu à un réseau de concepts liés. Le concept a pris racines dans l'excitation d'objets sonores avant de s'étendre vers les autres types d'objets et d'ondes. Mouhouche et El-Hajjami ont d'ailleurs élaboré une carte 
conceptuelle illustrant ce réseau (2010: 613). Cette carte résume le fonctionnement général, ou la phénoménologie, de la résonance et les liens qu'elle entretient avec d'autres concepts de physique. Trois notions y paraissent essentielles en ce qui a trait au phénomène de résonance survenant lors de l'excitation d'un résonateur par un excitateur en mouvement périodique:

- le couplage de l'excitateur et du résonateur à la fréquence propre du résonateur;

- le transfert d'énergie de l'excitateur vers le résonateur;

- et le bilan des énergies du résonateur, qui détermine l'issue de l'excitation résonante.

La résonance est l'effet ou le résultat, le phénomène est une excitation qui réunit les conditions de production d'une résonance. Le transfert d'énergie détermine ce qui se produit lors de cette excitation résonante. Son effet est capital. C'est grâce à lui que se transmettent les énergies des étoiles, celles des ondes radio que captent les récepteurs à transistors ainsi que toute énergie que transmet la matière (atome, molécule, etc.) et qui véhicule très souvent des informations. Le bilan des énergies (celle reçue et celle dissipée par le résonateur) explique l'évolution possible du système résonant. C'est ce bilan qui justifie l'atténuation, la permanence d'une résonance ou son évolution vers des situations potentiellement dangereuses par accumulation d'énergie.

C’est grâce aux notions de fréquence propre et de bilan d'énergie échangée par le résonateur que s'expliquent les multiples applications scientifiques (en technologie, en médecine, en chimie, en astronomie, etc.) de la résonance et de son exploitation dans les mesures, et que se définissent les diverses situations qui pourraient être à rechercher ou à éviter.

\section{Résultats}

Une mise en parallèle des évolutions terminologique et conceptuelle est réalisée dans le tableau 1 de synthèse joint en annexe. Ce tableau montre que résonance est un terme dont l'étymologie dérive du mot son et qu'il a été initialement utilisé pour exprimer la création ou le renforcement de sonorités, avant de désigner l'écho. Certains documents avancent même que son origine est plus précisément «re-sonner» (Nicot 1606: $563^{15}$ ). Nous sommes en présence d'un terme créé pour rendre compte d'un phénomène à l'origine naturel (observé dans la nature et audible). Partant de cette utilisation dans le langage courant, il fut d'abord utilisé dans un domaine restreint à l'acoustique pour se retrouver, actuellement, dans presque tous les domaines de la physique: mécanique, électricité, optique, électromagnétisme, acousto-optique, etc. En tant que concept physique, la résonance a connu une telle évolution qu'elle désigne aujourd'hui un phénomène omniprésent, touchant tous les types d'ondes et résultant d'un couplage de deux objets ou d'un couplage onde-objet, survenant à toutes les échelles, de celle des particules les plus élémentaires de la matière jusqu'à celle des planètes, dans le cas de la résonance orbitale. Le concept de résonance décrit ainsi un phénomène devenu complexe et général qui se produit dans des situations parfois insoupçonnées et s'étend sans cesse à de nouvelles interactions découvertes par la science au fil de l'évolution de celle-ci. Ce sont désormais des objets qui résonnent, et non l'onde ni la fréquence, la seconde étant un attribut essentiel de la première, ce que soutient le célèbre acousticien Hunt (1971: 435) en expliquant que 
«a frequency doesn't resonate». Dans la langue anglaise, cet auteur fait état de l'utilisation abusive de l'expression resonant frequency en lieu et place de resonance frequency pour faire référence à la résonance de l'onde, donnant lieu à une distorsion de sens et à des erreurs techniques. La première expression désigne la fréquence résonante alors que la deuxième signifie fréquence de résonance d'un objet.

Il apparaît, aussi bien dans l'étude métalexicographique que dans l'étude des manuels anciens de physique, que l'évolution sémantique du concept de résonance a connu trois phases principales au fil de l'histoire:

- Première signification: prolongement, réflexion d'un son et écho. Le domaine concerné était l'acoustique.

- Deuxième signification: influence d'un corps sonore sur un autre et transmission d'une vibration sonore. Les domaines concernés étaient: l'acoustique et la mécanique ondulatoire.

- Troisième signification: excitation à une fréquence propre d'un résonateur par un excitateur, ceux-ci étant constitués par des objets divers, avec transmission d'énergie. Il y a généralisation du concept et tous les domaines de la physique sont concernés.

Le terme résonance a donc acquis une signification qui n'est plus univoque. Cette polysémie rend difficile sa traduction fidèle et la compréhension de sa signification exacte, tout en générant des obstacles techniques et épistémologiques à l'apprentissage des notions qu'il désigne, dont notamment des obstacles cognitifs ${ }^{16}$.

Cette situation a des conséquences que nous examinons dans la section suivante, avant de proposer une remédiation possible pour la dénomination du phénomène de résonance.

\section{Conséquences didactiques et terminologiques}

L'écart observé entre un terme et l'évolution du concept qu'il désigne peut avoir des conséquences sur les plans didactique, épistémologique et traductologique, donc sur la perception du savoir et sur la construction des connaissances. Nous citons trois retombées pour ce qui est de la résonance.

\subsection{Obstacle verbal: "la résonance ne concerne que les ondes sonores»}

La polysémie peut notamment être à l'origine de ce que Bachelard appelle obstacle verbal en didactique des sciences (Bachelard 1938: 24). Ce point est également soulevé, entre autres auteurs, par Viennot (1979) et Astolfi (1997) en ce qui a trait aux sciences physiques, et par Iardella $(1998)^{17}$.

L'utilisation du terme résonance pour faire référence au son entravera, par exemple, la possibilité d'envisager des types de phénomènes résonants ayant lieu entre les planètes (résonance orbitale entre mouvements rotatoires). Les manuels d'enseignement évoquent très peu cette résonance, qui se trouve par conséquent écartée des cours de physique malgré son importance dans la recherche scientifique, notamment en astronomie. L'usage restreint du vocable résonance semble contribuer à faire croire que le phénomène qu'il désigne ne concerne que les ondes sonores, donc le domaine de l'acoustique, dans lequel il a été utilisé historiquement, alors qu'en réalité il s'applique à tous les types d'ondes, ne serait-ce qu'avec une signification physique appelée à varier. Il s'est opéré un changement dans le profil épistémologique dont 
Bachelard dit qu'il "garde la trace des obstacles qu'une culture a dû surmonter» (1940: 47) et qui induit un niveau de formulation et, donc, une représentation mentale qui sont également variables.

Cette difficulté peut se manifester dans les traductions et les transpositions et contribue à expliquer certaines confusions, notées dans nos observations de départ chez les étudiants et les enseignants, en ce qui a trait au niveau de formulation du concept.

\subsection{Difficulté à conceptualiser: "la résonance explique des faits perceptibles et décrit un comportement"}

L'histoire du concept de résonance a montré l'existence de plusieurs cas de résonance différant par la nature de l'excitateur ou du résonateur, ou par celle des ondes en jeu. Le phénomène, appelé à l'origine transmission des vibrations, désignait d'abord la vibration d'un corps, très souvent accompagnée d'une émission sonore (les corps étudiés étaient eux-mêmes appelés corps sonores). La résonance a maintenant un caractère beaucoup plus vaste. La difficulté à le conceptualiser, traduisant la difficulté de passage de la conception événementielle et substantialiste de la résonance vers une vision plus abstraite, n’a pu être franchie que lorsque le terme résonance s'est mis à désigner le phénomène vers la fin du XIX ${ }^{\mathrm{e}}$ siècle (Mouhouche et El-Hajjami 2010: 609). La résonance devint alors un concept abstrait et général. C’est ainsi que, depuis, il rend compte d'une réalité physique riche et diversifiée aux applications multiples. La conceptualisation est une opération mentale importante en science, elle fournit un moyen de lier les domaines et même les spécialités que la résonance concerne et d'opérer le transfert dans (et entre) les apprentissages.

La connotation sonore du terme résonance est réductrice et dépourvue de la dimension générale propre à la réalité du processus et à sa phénoménologie. Continuer à utiliser ce terme ferait croire que la résonance explique uniquement des faits perceptibles et décrit seulement un comportement seulement, car le signe linguistique est privé d'une partie de l'aspect interprétatif du savoir qu'il est censé véhiculer. C'est la raison pour laquelle ce signe ne favorise pas la conceptualisation. C'est celle-ci qui contribue à construire l'unité de compréhension dont parle Temmerman, avec sa représentation du savoir et son aspect interprétatif. Conceição écrit que:

[1]es termes sont des entités doubles dans lesquelles il nous faut considérer une face linguistique et une face cognitive. Le processus de dénomination est l'établissement d'une relation entre un ensemble de traits conceptuels et un ensemble de données linguistiques dans une langue. (Conceição 1999: 33)

Cette appellation de résonance à connotation sonore serait à l'origine de la difficile conceptualisation observée chez les étudiants, certains enseignants et certains auteurs, ce pourquoi la face cognitive ne correspond pas (en fait, ne correspond plus) à la face linguistique du terme. 


\subsection{Traduction de textes scientifiques}

Dury $(1999: 494,495)$ s'est exprimée sur la nécessité qu'il y a, pour le traducteur, de vivre en bonne intelligence avec le concept qu'il traduit et, pour le terminologue, de mener une réflexion adaptée pour créer des outils d'aide à la traduction. Concernant la nécessité qu'ils ont de connaître le domaine d'origine du concept, les diverses dénominations qui l'ont désigné, l'évolution historique qu'il a connue et sa définition actuelle, elle affirme que

ces préalables permettent indéniablement de mieux connaître le concept et donc de le traduire dans de meilleures conditions, en s'éloignant d'une traduction littérale du signe qui, lorsqu'elle est systématiquement appliquée, peut conduire à des équivalences médiocres. (Dury 1999: 494)

L'auteure conclut que les traducteurs, selon qu'ils sont spécialistes ou non spécialistes, n'ont pas besoin des mêmes outils terminographiques. La mise au point d'outils d'aide à la traduction, différents pour ces deux catégories de traducteurs, serait même une piste de recherche nouvelle en terminologie.

Notre étude montre que le terme résonance exprime plusieurs réalités. Même accompagné de son contexte, il risque d'être mal traduit vers d'autres langues, notamment celles qui consacrent un terme différent pour chaque contexte. La langue anglaise utilise resonance et resounding pour désigner ce phénomène, et différents verbes pour résonner: to resound, to echo et to reverberate pour un phénomène sonore (retentissement, écho, etc.) et to resonate pour un phénomène d'excitation d'un élément par un autre.

La langue arabe utilise deux termes distincts, correspondants aux deux cas précédents, pour traduire résonance (Mouhouche 1989/2004) ${ }^{18,19}$, et des confusions existent dans les manuels quant à l'utilisation de ces termes.

Le tableau 2 synthétise ces traductions et fait apparaître parallèlement les correspondances possibles dans le passage d'une langue à une autre. Des difficultés de compréhension pourraient alors exister du fait de l'influence du langage, en tant qu'instrument cognitif, sur le processus de compréhension (Temmerman 2000a).

Renommer le phénomène scientifique de résonance peut permettre de prévenir les confusions préjudiciables à la compréhension et à la précision dans les traductions ou la conception de documents et de ressources scientifiques. La section qui suit donne des propositions en ce sens.

\section{Propositions terminologiques et pédagogiques}

Pour faciliter aussi bien la traduction que l'apprentissage, nous pensons qu'il est judicieux de sortir de l'influence sonore de la racine étymologique des termes résonner et résonance. Cette racine (son) est liée au sens qu'avait le terme résonance avant le milieu du XviII ${ }^{\mathrm{e}}$ siècle. De plus, le verbe résonner confère à l'objet qui résonne, le résonateur, un rôle actif: il aurait la capacité d'agir (résonner), alors qu'en réalité il est mis en résonance, puisqu'il est excité par un autre objet, l'excitateur, dans le sens physique. Ce phénomène pourrait être à l'origine d'autres formes d'obstacles épistémologiques ${ }^{20}$.

Il serait préférable d'utiliser un terme ou une expression plus proche de la réalité des faits et qui rend compte du concept scientifique tel qu'il doit être appréhendé par 
l'étudiant ou par un lecteur de document scientifique spécialisé. Au lieu de résonance, nous proposons l'utilisation du syntagme excitation résonante. Ce syntagme a l'avantage d'exprimer, grâce au terme excitation, l'intervention d'un excitateur sur un résonateur, ce qui se produit lors d'un phénomène de résonance. Cela est exprimé par l'idée de transmission ou de communication de vibrations quavait introduite Jamin (1887, voir section 3.2.1.2.) dans son manuel de physique. Ce terme (excitation) a aussi bien été utilisé dans la définition du phénomène de résonance à partir de 1765 d'après le FEW (Wartburg 1948: 305b), qui parle de corps «soumis à une excitation convenable», ou dans l'expression de vibration excitatrice (section 3.1). Il rend donc plus explicite ce que l'on veut désigner dans le domaine scientifique.

L'adjectif résonante, adjoint à excitation dans le syntagme proposé, conserve quant à lui l'idée de se placer dans une situation de résonance. Ce syntagme peut exprimer toutes les résonances (même celles se produisant par ondes sonores) en tant que transfert d'énergie d'un excitateur vers un résonateur à la fréquence propre à ce dernier.

Dans un texte spécialisé en physique, l'expression excitation résonante exprimant l'action d'un objet excitateur sur un objet excité, lorsqu'elle est traduite d'une langue vers une autre, oblige à rendre compte de ce rapport physique et phénoménologique entre objets vibrants et ne laisse pas place à d'autres interprétations. D’autant plus que le terme excitation exprime le transfert d'énergie, aspect physique qui, comme nous l'avons écrit précédemment, est primordial dans tout processus de résonance ${ }^{21}$.

Par ailleurs, nous pensons que l'introduction dans l'enseignement, et donc dans les manuels de physique, d'éléments d'histoire du concept apporterait un supplément d'éclairage et d'intérêt pour son apprentissage. Ce regard terminologique diachronique pourrait d'ailleurs être inclus dans un cours d'histoire de la physique, ou même d'histoire des sciences, afin d'élargir la compréhension des problématiques abordées. Des physiciens tels Pietschmann ont déjà soutenu que certains sujets de physique et des questions des plus complexes pourraient s'avérer plus facilement accessibles grâce à une optique diachronique (Celotti et Musacchio 2004).

\section{Conclusion}

L'étude diachronique du concept de résonance est riche en enseignements. Elle a fait apparaître, à travers le parallélisme entre ses évolutions terminologique et conceptuelle, que le concept scientifique a évolué et changé de signification sans modification dans la dénomination. Le terme de résonance, exprimant à l'origine un phénomène naturel ressenti surtout par l'ouïe, s'est d'abord limité au domaine de l'acoustique, dont il garde l'estampille jusqu'à maintenant. La résonance est devenue par la suite un concept scientifique d'une grande importance en physique, qui s'est élargi pour englober tous les types d'ondes, mêmes invisibles et inaudibles, et inclure diverses dimensions de la nature, des particules élémentaires de la matière aux planètes, en passant par les marées. Ce concept s'est mis à désigner des phénomènes étendus à des objets résonants de plus en plus diversifiés, donc à s'appliquer à d'autres situations parfois très éloignées de l'univers sonore à strictement parler ${ }^{22}$. De plus, il véhicule les idées d'accord et de transfert d'énergie, inexistantes à l'origine et absentes du sens qui lui est actuellement donné dans le langage courant. L'apparition de ces nouvelles réalités scientifiques sans qu'il n'y ait de changement de dénomination rend le terme 
résonance polysémique. Bouasse avait entrevu cette réalité lorsqu'il a écrit que: «Les noms, à peu près tous, ont été mal choisis, parce qu'ils l'ont été quand on n'avait qu'une notion imparfaite des phénomènes à nommer» (1909: 62). L'existence de cette polysémie, dans le cadre des sciences physiques, est à l'origine d'ambiguïtés dans les traductions et de certains obstacles cognitifs et épistémologiques.

Une dénomination plus adaptée à ce que les physiciens entendent par le terme résonance actuellement est proposée. Le syntagme excitation résonante nous parait mieux exprimer l'influence d'un excitateur sur un résonateur, avec transfert d'énergie, conséquent à un accord résonant des fréquences. La notion d'excitation était apparue au milieu du XviII ${ }^{e}$ siècle dans les définitions du phénomène de résonance.

La présente étude pourrait avoir plusieurs retombées. Tout en donnant un point de vue complémentaire, elle apporte un éclairage important à l'analyse historique et épistémologique de la résonance en physique des ondes. Elle pourrait contenir des éléments susceptibles de contribuer à l'élaboration d'outils d'aide à la traduction. Elle contribue parallèlement à montrer l'intérêt de l'analyse diachronique pour la transposition didactique, la traduction de manuels et l'amélioration de l'enseignementapprentissage du concept.

\section{NOTES}

1. Nous considérons les deux termes (notion et concept) comme équivalents, conformément aux recommandations de l'Association française de normalisation (AFNOR).

2. Nicot, Jean (1606): Thresor de la langue françoyse. Paris: David Douceur: Consulté le 26 août 2011, $<$ http://portail.atilf.fr/>.

3. Dictionnaire de l'Académie Française, $1^{\mathrm{re}}$ éd. (1694): Paris: Chez la Veuve de Jean Baptiste Coignard.

4. Brachet, Auguste (1870): Dictionnaire étymologique de la langue française. Paris: Hetzel et $C^{\text {ie. }}$.

5. Trésor de la langue française informatisé (TLFi) (Dernière mise à jour: 2012): Consulté le 2 octobre 2011, <http://www.cnrtl.fr>.

6. WARTbURG, Walther von (1948): Französisches Etymologisches Wörterbuch (FEW). Vol. 10. Bâle: Zbinden Druck und Verlag AG.

7. Nouveau Petit Robert 2.1 (2001) [cédérom] : Paris: Dictionnaires Le Robert.

8. Oxford Dictionary (Dernière mise à jour : 2012): Oxford: Oxford University Press: Consulté le 15 janvier 2012, <http://oxforddictionaries.com>.

9. Collins French-English Dictionary et Collins English Dictionary (Dernière mise à jour: 2012): Consultés le 15 janvier 2012, <http://www.collinsdictionary.com>.

10. Nous utilisons cette caractéristique de «sonorisation» pour exprimer le rapport au son du terme ou du concept de résonance. C'est cette «sonorisation» qui posera problème plus tard.

11. Il est bien connu maintenant que l'onde sonore fait vibrer la structure de l'oreille interne dotée de plusieurs fréquences de résonance et que l'oreille interne achemine au cerveau l'information auditive codée selon la fréquence et l'intensité (Giancoli 1993: 26). De même d'ailleurs que l'onde lumineuse est perçue de façon similaire par l'œil, dont la rétine comporte elle aussi des structures dotées de fréquences de résonance (Giancoli 1993: 126).

12. Brenet, Michel (1926) : Dictionnaire pratique et historique de la musique. Paris: Armand Colin.

13. En physique, l'absorption et l'émission de radiofréquences dues à l'oscillation et à la réorientation des spins nucléaires en présence de champs magnétiques externes ou internes sont caractérisées comme étant une forme de résonance au même titre, par ailleurs, que les radiations émises par des atomes revenant à l'état fondamental après avoir été portés à des états de plus grande énergie.

14. Étudiées par Helmholtz, Hertz et Laplace, ces résonances sont peu transposées dans les programmes et dans les manuels, en dépit de leur importance. Le passage suivant, tiré d'Encrenaz, Barucci, et al. (2003: 12), donne un aperçu de celle-ci: «Les résonances peuvent lier entre eux plus de deux corps. Des résonances peuvent aussi lier entre eux plusieurs paramètres d'orbites, par exemple le moyen mouvement du satellite et la vitesse de rotation de la planète; un exemple spectaculaire en est la rotation synchrone des satellites et en particulier de la Lune (résonance spin: orbite $1: 1)$ : La période de rotation de la lune autour de la Terre est égale à la période de rotation 
sur elle-même, ce qui implique qu'elle présente toujours la même face à la Terre. Les frottements suscités dans le satellite par les forces des marées [...] ont ralenti celui-ci jusqu'à la rotation synchrone où les frottements disparaissent. Tous les satellites réguliers du système solaire sont en rotation synchrone avec leur planète.»

15. Nicot, dans le Thresor de la langue françoyse, écrit «resonner» pour «sonner de rechef» (1606: 563).

16. Les obstacles cognitifs peuvent être d'ordre épistémologique, didactique, ontologique ou même psychologique (Bachelard 1938: 14).

17. «Les mots peuvent constituer de véritables obstacles verbaux pour le scientifique, car celui-ci peut se retrouver pris au piège de certaines images qui, par la métaphore suggérée, poussent la pensée dans le règne de l'image, mettant en jeu des qualités substantielles, qui sont un frein au raisonnement scientifique», écrit Iardella (1998: 87).

18. Mounouche, Ali (1988/1994): Lexique de vocabulaire de physique, français-arabe. Alger: O.P.U.

19. En langue arabe il existe le terme tadjawoub, bien adapté pour désigner le phénomène physique général de résonance, mais il est très souvent traduit par ranine ou tanine, qui font eux référence au bruit et aux sons, équivalents de résonance sonore.

20. Nous pensons à l'obstacle caractérisé d'anthropomorphique qui a été décrit par Bachelard (1938) comme étant un obstacle épistémologique consistant en l'attribution de qualités humaines aux phénomènes physiques ou biologiques. Cet obstacle est caractéristique de la pensée pré-scientifique, issue particulièrement des $\mathrm{XVII}^{\mathrm{e}}$ et $\mathrm{XVIII}^{\mathrm{e}}$ siècles. Cette projection de comportements humains sur les phénomènes est un obstacle majeur que le scientifique doit savoir déjouer (Iardella 1998: 87). La désignation des objets émettant un son par les expressions corps sonore et corde excitée, ainsi que les écrits de Helmholtz voulant qu'une corde parle, sont des signes de cet obstacle.

21. Nous pouvons avancer, d'après la carte conceptuelle de la résonance (Mouhouche et El-Hajjami 2010: 613), que la résonance est avant tout un transfert d'énergie qui, avec les dissipations du résonateur, régit tout le processus et l'évolution du phénomène. L’augmentation de l'amplitude du résonateur n'est qu'une conséquence de ce transfert d'énergie.

22. En matière d'explication paradigmatique, ce changement montre également le passage d'une conception mécaniste, expliquant les phénomènes par la causalité, à une conception réaliste intégrant la complexité et la diversité du phénomène. Ce changement est en rapport avec les progrès de la physique, notamment en ce qui concerne la matière et la découverte des propriétés des particules.

\section{RÉFÉRENCES}

Antoine, M.J. (1849): Résonnance multiple et phénomènes optiques produits par les corps vibrants. Annales de chimie et de physique, troisième série, tome vingt-septième. Paris: Victor Masson, 191-209. Consulté le 21 février 2011, <http://books.google.dz/books?id= Eg8AAAAAMAAJ\&hl $=$ fr $>$.

Astolfi, Jean-Pierre (1997): L'erreur, un outil pour enseigner. Paris: E.S.F.

BACHELARD, Gaston (1938): La formation de l'esprit scientifique. Paris: Vrin.

Bachelard, Gaston (1940): La philosophie du non. Paris: PUF.

Blaserna, Pietro (1877): Le son et la musique. Paris: Librairie Germer Baillière et Cie.

Bouasse, Henry (1909): Service des renseignements: question 152. Bulletin de l'Union des Physiciens. 4(28):61-64.

Boutet de Monvel, Benjamin (1863): Cours de physique. Paris: L. Hachette et Cie.

Celotti, Nadine et Musacchio, Maria Teresa (2004): Un regard diachronique en didactique des langues de spécialité. Revue de didactologie des langues-cultures et de lexiculturologie. 135(3):263-270.

Chappuis, James et Berget, Alphonse (1892): Leçons de physique générale (tome III). Paris: Gauthier-Villars.

Chappuis, James et Lamotte, Marcel (1911): Leçons de physique générale (tome IV). Paris: Gauthier-Villars et Cie.

Clas, André (2001) : Compte-rendu de Temmerman, Rita (2000) : Towards New Ways of Terminology Description. The Sociocognitive Approach. Amsterdam/Philadelphia, John Benjamins. Meta: journal des traducteurs. 46(3):582-585. 
ConCEIÇão, Manuel Célio (1999): Terminologie et transmission du savoir: (re)construction(s) de concepts. In: Valérie Delavigne et Myriam Bouveret, dir. Sémantique des termes spécialisés. Havre: Publication de l'Université de Rouen, 33-42.

ConceIção, Manuel Célio (2005): Concepts, termes et reformulations. Lyon: Presses Universitaires de Lyon.

Daguin, Pierre-Adolphe (1861-1862): Traité élémentaire de physique théorique et expérimentale. Toulouse: E. Privat.

Drude, Paul (1911): Précis d'optique, tome 1 et tome 2. Paris: Gauthier-Villars.

DurY, Pascaline (1999): Étude comparative et diachronique des concepts ecosystem et écosystème. Meta: Journal des traducteurs. 44(3):485-499.

Dury, Pascaline (2006): La dimension diachronique en terminologie et en traduction spécialisée: cas de l'écologie. In: Danielle CANDel et François Gaudin, dir. Aspects diachroniques du vocabulaire. Havre: Publication de l'Université de Rouen, 109-124.

Encrenaz, Thérèse, Barucci, Maria-Antonietta, Bibring, Jean-Pierre, et al. (2003): Le système solaire. Paris: EDP Sciences et CNRS Éditions.

FeLber, Helmut (1987): Manuel de terminologie. Paris: Unesco, Infoterm.

Fournié, Edouard (1866): Physiologie de la voix et de la parole. Paris: Adrien Delahaye.

Giancoli, Douglas C. (1993): Physique générale 3 (Ondes, optique et physique moderne). Bruxelles: De Boeck Université.

Hunt, Frédéric Vinton (1971): Resonance versus Resonant. The Journal of the Acoustical Society of America. 50(2):435-435.

IARDELla, Emmanuelle (1998): Sciences humaines et sociales. Paris: Masson.

Jamin, Jules (1887): Cours de physique de l'école polytechnique (tome 3). Paris: Gauthier-Villars.

Kunn, Thomas (2001): La structure des révolutions scientifiques. Manchecourt: Champs.

Madrane, Mourad, Talbi, Mohammed, Khaldi, Mohamed, et al. (2008): Des implications didactiques possibles de l'histoire de la théorie cellulaire dans une optique de formation à l'enseignement des sciences. RADISMA. 3. Consulté le 27 mars 2001, <http://www.radisma. info/document.php?id=603>.

Mounouche, Ali et El-HajJami, Abdelkrim (2010): Étude de la resonance: quels obstacles épistémologiques. Bulletin de l'Union des Physiciens. 104(924):599-613.

Peclet, Eugène (1847): Traité élémentaire de physique (Tome 1). $4^{\mathrm{e}}$ éd. Paris: L. Hachette et Cie.

Temmerman, Rita (1998a): Terminology Beyond Standardisation. Language and Categorisation in the Life Sciences. Thèse de doctorat non publiée. Louvain: Université catholique de Louvain.

Temmerman, Rita (1998b): Why traditional terminology theory impedes a realistic description of categories and terms in the life sciences. Terminology. 5(1):77-92.

Temmerman, Rita (2000a): Une théorie réaliste de la terminologie: le sociocognitivisme. Terminologies nouvelles. $21: 58-64$.

Temmerman, Rita (2000b): Towards New Ways of Terminology Description. The Sociocognitive Approach. Amsterdam/Philadelphie: John Benjamins.

VIENNOT, Laurence (1979): Le raisonnement spontané en dynamique élémentaire. Paris: Hermann.

WÜSTER, Eugen (1991): Einführung in die allgemeine Terminologielehre und terminologische Lexikographie, 3. Aufl. Bonn: Romanistischer Verlag. 


\section{ANNEXES}

TABLEAU 1

Tableau synthétique: mise en parallèle de la terminologie et des significations et des applications du concept dans l'histoire

\begin{tabular}{|c|c|c|}
\hline Dates & Terminologie & Significations et applications \\
\hline $\begin{array}{l}1155 \text { ou } \\
1160\end{array}$ & $\begin{array}{l}\text { Apparition du verbe } \\
\text { résonner }\end{array}$ & $\begin{array}{l}\text { Produire un son accompagné de résonances; retentir en } \\
\text { s'accompagnant de résonances; renvoyer un écho; retentir (du } \\
\text { bruit de...); s'emplir de bruit, d'échos, de résonances (lieu). }\end{array}$ \\
\hline $\begin{array}{l}1365 \text { ou } \\
1374\end{array}$ & $\begin{array}{l}\text { Apparition du terme } \\
\text { résonance }\end{array}$ & $\begin{array}{l}\text { Prolongation de la durée d'un son, prolongement ou amplification } \\
\text { des sons dans certains milieux sonores, renforcement de } \\
\text { l'intensité vibratoire ou sonore. }\end{array}$ \\
\hline 1538 & résonant & Qui est le siège d’un phénomène de résonance. \\
\hline 1746 & résonance & Propriété d'un corps d'entrer en vibration. \\
\hline 1863 & résonance & $\begin{array}{l}\text { Réflexion d'un son. } \\
\text { Echo (si la réflexion se produit sur un obstacle éloigné). }\end{array}$ \\
\hline 1862 & $\begin{array}{l}\text { Modification de la } \\
\text { signification de résonance }\end{array}$ & $\begin{array}{l}\text { Transmission de vibration par l'air. } \\
\text { Augmentation de l'amplitude d'un système physique en vibration } \\
\text { lorsque la vibration excitatrice se rapproche d'une fréquence } \\
\text { naturelle de ce système. }\end{array}$ \\
\hline 1868 & $\begin{array}{l}\text { résonateur (utilisation en } \\
\text { science) }\end{array}$ & $\begin{array}{l}\text { Appareil où peut se produire une résonance, un milieu matériel } \\
\text { capable d'entrer en vibration sous l'influence d'un excitateur. }\end{array}$ \\
\hline 1887 & $\begin{array}{l}\text { résonance acoustique et } \\
\text { autre qu'acoustique }\end{array}$ & $\begin{array}{l}\text { La résonance acoustique est appelée renforcement des sons. } \\
\text { Le phénomène de résonance autre qu'acoustique est décrit par } \\
\text { l'expression transmission ou communication de vibrations. }\end{array}$ \\
\hline 1911 & $\begin{array}{l}\text { Généralisation de la } \\
\text { signification de résonance }\end{array}$ & $\begin{array}{l}\text { Chappuis et Lamotte utilisent le terme résonance pour les ondes } \\
\text { électriques, et parlent de l'expérience de Hertz, dans le sens admis } \\
\text { maintenant. }\end{array}$ \\
\hline 1911 & résonance optique & $\begin{array}{l}\text { Paul Drude explique la modification de la lumière, produite par } \\
\text { les milieux troubles, par l'absorption d'un rayonnement par } \\
\text { résonance et donne des résultats qu'il décrit comme «absolument } \\
\text { conformes aux phénomènes généraux de résonance». }\end{array}$ \\
\hline 1926 & $\begin{array}{l}\text { résonance en mécanique } \\
\text { quantique }\end{array}$ & $\begin{array}{l}\text { Heisenberg, en 1926, utilise le mot résonance pour la première fois } \\
\text { en mécanique quantique dans ses travaux sur les systèmes } \\
\text { d'oscillateurs couplés. }\end{array}$ \\
\hline 1930 & résonance en chimie & $\begin{array}{l}\text { Introduite par Pauling dans ses recherches sur la nature de la } \\
\text { liaison chimique. }\end{array}$ \\
\hline
\end{tabular}

\section{TABLEAU 2}

Traductions en anglais et en arabe de résonner et résonance

\begin{tabular}{|c|c|c|c|}
\hline Français & Sens & Anglais & Arabe \\
\hline \multirow{4}{*}{ Résonner } & Retentir (son), faire écho & \multirow{2}{*}{$\begin{array}{l}\text { to resound, } \\
\text { to reverberate, } \\
\text { to echo }\end{array}$} & \multirow{2}{*}{$\begin{array}{l}\text { yarennou, } \\
\text { yatennou }\end{array}$} \\
\hline & Emettre un son par excitation acoustique & & \\
\hline & \multirow{2}{*}{$\begin{array}{l}\text { Vibrer par communication de vibrations ou par } \\
\text { absorption de rayonnement }\end{array}$} & \multirow{2}{*}{$\begin{array}{l}\text { to resonate, } \\
\text { to echo }\end{array}$} & $\begin{array}{l}\text { yarennou, } \\
\text { yatennou, }\end{array}$ \\
\hline & & & yatadjawebou \\
\hline \multirow{3}{*}{ Résonance } & Retentissement, écho & \multirow{3}{*}{$\begin{array}{l}\text { resonance, } \\
\text { resounding, } \\
\text { echo }\end{array}$} & \multirow[b]{2}{*}{$\begin{array}{l}\text { ranine, } \\
\text { tanine }\end{array}$} \\
\hline & $\begin{array}{l}\text { Augmentation de vibration sonore par absorption } \\
\text { d'énergie acoustique }\end{array}$ & & \\
\hline & $\begin{array}{l}\text { Acquisition d'énergie par excitation résonante ou } \\
\text { absorption de rayonnement }\end{array}$ & & $\begin{array}{l}\text { ranine, tanine, } \\
\text { tadjawoub }\end{array}$ \\
\hline
\end{tabular}

